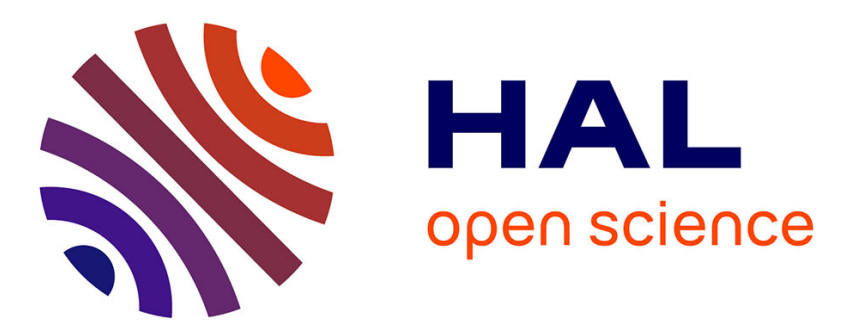

\title{
Collective surface modes in small spherical metallic systems within the Bloch-Jensen hydrodynamical model
}

J. Kupersztych, Michèle Raynaud

\section{To cite this version:}

J. Kupersztych, Michèle Raynaud. Collective surface modes in small spherical metallic systems within the Bloch-Jensen hydrodynamical model. Journal of Physics: Condensed Matter, 1994, 6, pp.10669 10682. 10.1088/0953-8984/6/49/010 . hal-01949737

\section{HAL Id: hal-01949737 \\ https://hal.science/hal-01949737}

Submitted on 26 Dec 2020

HAL is a multi-disciplinary open access archive for the deposit and dissemination of scientific research documents, whether they are published or not. The documents may come from teaching and research institutions in France or abroad, or from public or private research centers.
L'archive ouverte pluridisciplinaire HAL, est destinée au dépôt et à la diffusion de documents scientifiques de niveau recherche, publiés ou non, émanant des établissements d'enseignement et de recherche français ou étrangers, des laboratoires publics ou privés. 


\section{Collective surface modes in small spherical metallic systems within the Bloch-Jensen hydrodynamical model}

To cite this article: J Kupersztych and M Raynaud 1994 J. Phys.: Condens. Matter 610669

View the article online for updates and enhancements.

\section{Related content}

- Dispersion of plasmons in metallic
spheres: dipole mode
N Barberan and M Batlle
- Interface plasmons at metal-metal
$\frac{\text { junctions }}{\text { P Jewsbury }}$
- Theory of surface plasmons and surface-
plasmon polaritons
J M Pitarke, V M Silkin, E V Chulkov et al.

\section{Recent citations}

- Plasmon Spectra of Cylindrical
Nanostructures Including Nonlocal Effects
Afshin Moradi and Elham Ebrahimi
- Linear and nonlinear light scattering and
absorption in free-electron nanoclusters
with diffuse surface: General
Considerations and linear response
S. V. Fomichev and W. Becker

\section{IOP ebooks'}

Bringing you innovative digital publishing with leading voices

to create your essential collection of books in STEM research.

Start exploring the collection - download the first chapter of every title for free. 


\title{
Collective surface modes in small spherical metallic systems within the Bloch-Jensen hydrodynamical model
}

\author{
J Kupersztych and M Raynaud \\ Commissariat à I'Energie Atomique, DRECAM/SRSIM, Bâtiment 462, Centre d'Etudes de \\ Saclay, 91191 Gif-sur-Yvette Cédex, France
}

Received 29 June 1994, in final form 26 September 1994

\begin{abstract}
An exact generalization of the classical Bloch-Jensen hydrodynamical model for the homogeneous free-electron gas is obtained for a two-layer spherical metal system. The dispersion relation of surface plasmons together with the analytical expression of the selfconsistent electric field are calculated exactly. It is found that small and large systems exhibit notably different behaviours. In particular, the surface-plasmon frequencies strongly depend on the layer thickness. As is the case for the metal-vacuum interface, the electric field is shown to present a sharp maximum at the interface between the two metals. A simple physical interpretation of this effect is provided. The present two-layer system also gives interesting indications about the part played in collective surface modes by the ion-density profile near the surface of small metal clusters, which may be helpful in interpreting recent puzzling experimental data.
\end{abstract}

\section{Introduction}

The interest in surface plasmons (SPs) has been recently revived, particularly in relation to fragmentation measurements of small metallic clusters. For instance, recent experiments have revealed that clusters of a small number of alkali atoms (less than $10^{3}$ ) are characterized by unexpected SP frequencies (De Heer 1993, Bréchignac et al 1993). Moreover, SPs have been shown to exist even for systems involving very few atoms (five to 20) (Parks and McDonald 1989, Wang et al 1993, De Heer 1993). In this context, several questions have been raised. Some authors have discussed the influence of size effects or spill-out of the electron distribution at the surface on the SP frequencies (Xu and Dignam 1992, Lipparini and Pederiva 1993, Malov and Zartsky 1993, Brack 1993). However, to our knowledge, the role of ion-density inhomogeneity near the surface has not been clearly investigated, while it is expected to play a role, particularly for small systems such as small metal clusters. This issue is the main purpose of this paper.

At a microscopic level, the full treatment of an ion density gradient is an extremely difficult task. In this paper, we will use the following alternative macroscopic approach. To take inhomogeneous ion-density effects into consideration, a simple device consists in representing the surface region as a sequence of homogeneous layers of decreasing ion density (Boardman and Paranjape 1977, Boardman 1982). This method clearly amounts to solving the cumbersome problem of a metal bulk covered by a multiple-layer metal system. On the other hand, its advantage is that it enables us to represent each metal layer as a jellium, thus making the use of Bloch's hydrodynamical model (1933) for a homogeneous freeelectron gas possible and deriving benefit from the exact solution for a single homogeneous medium obtained long ago by Jensen (1937). Obviously, the hydrodynamical model is 
valid provided that the collective aspects of the electron gas prevail over the collisional ones. This condition will be fulfilled when the frequency of the collective oscillations is much higher than the electron-ion (Drude's inverse lifetime) and electron-electron collision frequencies. In general, for a medium to be correctly described as a collisionless plasma, i.e. to neglect electron-electron collisions, the mean free path $\ell$ should be large compared with the wavelength $\lambda$ of the macroscopic self-consistent electric field. For free-electron metals, the Drude inverse lifetime, the electron-electron collision frequency $\nu_{e-e}$ and the plasma frequency $\omega_{\mathrm{p}}$ are, respectively, of the order of $10^{14} \mathrm{~s}^{-1}, 10^{10} \mathrm{~s}^{-1}$ (Ashcroft and Mermin 1976) and $10^{16} \mathrm{rad} \mathrm{s}^{-1}$. Also, we have $\ell \approx v_{\mathrm{F}} / \nu_{\mathrm{e}-\mathrm{e}}$ (where $v_{\mathrm{F}}$ denotes the Fermi velocity), which is of the order of $10^{-2} \mathrm{~cm}$. Thus, SP modes having oscillation frequencies of the order of $\omega_{\mathrm{p}} / \sqrt{3}$ may be reasonably described within this model, provided appropriate conditions taking account of specific (kinetic) effects in the high-frequency regime are laid down (see below). This explains why the Bloch-Jensen hydrodynamical model has been successful for simple metals (Lundqvist 1983) in spite of its limitations. In addition, this approach has the advantage of analytical tractability.

In this paper, we wish to show that an exact analytical generalization of Jensen's work can be built for a spherical metallic two-layer system, thus giving the exact dispersion relation of SPS modified by the presence of a thin metal layer. Our two-layer system will also give useful indications about the role played by the surface-density profile in simple metal clusters. Let us emphasize that this problem is not exactly equivalent to that of an inhomogeneous electron sphere such as considered long ago by Ruppin (1976) and by Boardman and Paranjape (1977), which is not truly a two-jellium system and which therefore requires different boundary conditions. Finally, our simple model will be proven useful to show an interesting effect: the existence of an interface plasmon at the metal-metal interface enhanced by the field of the SP.

\section{The Bloch-Jensen hydrodynamical model for a two-layer system}

In the Bloch-Jensen hydrodynamical formalism, the collective oscillations of a homogeneous electron gas are described by Poisson's equation

$$
\nabla \cdot \boldsymbol{E}(r, t)=4 \pi e\left[n_{\mathrm{ion}}-n(r, t)\right]
$$

the charge conservation equation

$$
\partial n(r, t) / \partial t+\nabla \cdot[n(r, t) v(r, t)]=0
$$

and the fluid equation of motion

$$
\partial v(r, t) / \partial t+\frac{1}{2} \nabla v^{2}(r, t)=-e E(r, t) / m-\nabla P(r, t) / m n(r, t) .
$$

In these equations, $E$ is the self-consistent electric field, $v$ the fluid velocity and $P$ the kinetic pressure. $n_{\text {ion }}$ and $n$ denote respectively the ion and electron densities.

In the case of a two-layer metal system described within the framework of a jellium model (electron densities are neutralized everywhere by uniform background ion densities) that consists of a spherical bulk (medium (I)) of radius $R$ and electron density $n_{0}^{(1)}$ overlaid by a foil (medium (II)) of thickness $h$ and electron density $n_{0}^{(\mathrm{II})}$, there are two electronand ion-density singularities: one between the two metallic media and another between metal and vacuum. Now, it can be easily demonstrated (see the appendix) that the above 
equations (1)-(3) still hold provided continuity of the normal (radial) component of the electron current is prescribed everywhere. Furthermore, since at the interface $(r=R)$ between medium (I) and medium (II) we deal with two perfect conductors in interaction, the other boundary conditions include continuity of all the components of the electric field:

$$
\left[E^{(i)}(r, t)\right]_{r=R}=\left[E^{(\mathrm{II})}(r, t)\right]_{r=R}
$$

and continuity of all the components of the electron current (see the appendix): $j(r, t)=$ $-e n(r, t) v(r, t)$

$$
\left[j^{(\mathrm{I})}(r, t)\right]_{r=R}=\left[j^{(\mathrm{II})}(r, t)\right]_{\mathrm{r}=R} .
$$

At $r=R+h$, as in the Jensen case, we deal with the interface between a perfect conductor (medium (II)) and a perfect insulator (vacuum). The corresponding boundary conditions (Jackson 1976) are continuity of all the components of the electric field

$$
\left[E^{(\mathrm{II})}(r, t)\right]_{r=R+h}=\left[E^{(\mathrm{vac})}(r, t)\right]_{r=R+h}
$$

and continuity of the sole radial component of the electron current (see the appendix)

$$
\left[j_{r}^{(\mathrm{II})}(r, t)\right]_{r=R+h}=0 .
$$

\section{The linear electron response}

The following theoretical treatment of (1)-(3) is standard inasmuch as we are only interested in the linear electron response. (1)-(3) are thus linearized by writing

$$
n^{(i)}(r, t)=n_{0}^{(i)}+n^{(i)}(r) \mathrm{e}^{-\mathrm{i} \omega t}
$$

with $\left|n^{(i)}\right| \ll n_{0}^{(i)}$, where $i=\mathrm{I}$ and II. As above mentioned, in the jellium model, the ion density is assumed to neutralize the electron density at equilibrium, that is, $n_{\text {ion }}^{(i)}=n_{0}^{(i)}$. By means of this procedure, (1)-(3) take the following form:

$$
\begin{aligned}
& \nabla \cdot E^{(i)}(r)=-4 \pi e n^{(i)}(r) \\
& -\mathrm{i} \omega n^{(i)}(r)+\nabla \cdot\left[n_{0}^{(i)} v^{(i)}(r)\right]=0 \\
& \mathrm{i} \omega n_{0}^{(i)} v^{(i)}(r)=e n_{0}^{(i)} E^{(i)}(r) / m+\nabla P^{(i)}(r) / m .
\end{aligned}
$$

According to Bloch and Jensen, we take the kinetic pressure in the form

$$
P^{(i)}(r)=m \beta^{(i) 2} n^{(i)}(r)
$$

where, for the hydrodynamical approach to be valid in the high-frequency regime, we have to assume (Pines 1964) $\beta^{(i)}=\sqrt{\frac{3}{5}} v_{\mathrm{F}}^{(i)}$, where $v_{\mathrm{F}}^{(i)}=\left(3 \pi^{2} n_{0}^{(l)}\right)^{1 / 3} \hbar / m$ is the Fermi velocity of the electron gas.

The above equations (8)-(10) will take a particularly simple form when introducing the scalar potential $\Phi(r)$ of the electric field $[E(r)=-\nabla \Phi(r)]$. Thus, if $k^{(i)}=$ 
$\left(\omega^{2}-\omega_{\mathrm{p}}^{(i) 2}\right)^{1 / 2} / \beta^{(i)}$ where $\omega_{\mathrm{p}}^{(i)}=\left(4 \pi e^{2} n_{0}^{(i)} / m\right)^{1 / 2}$ is the plasma frequency, they reduce to the following equations:

$$
\left[\Delta+k^{(i) 2}\right] \Delta \Phi^{(i)}(r)=0
$$

for the material medium, and for vacuum

$$
\Delta \Phi^{\mathrm{vac}}(\boldsymbol{r})=0 .
$$

In spherical coordinates, the general form of the solution of (11) is known as (Courant and Hilbert 1989)

$$
\Phi^{(i)}(r)=\sum_{\ell, m} f_{\ell m}^{(i)}(r) Y_{\ell m}(\theta, \varphi)
$$

with

$$
f_{\ell m}^{(i)}(r)=A_{\ell m}^{(i)} r^{\ell}+B_{\ell m}^{(i)} r^{-(\ell+1)}+D_{\ell m}^{(i)} j_{\ell}\left(k^{(i)} r\right)+F_{\ell m}^{(i)} y_{\ell}\left(k^{(i)} r\right)
$$

and for (12)

$$
\Phi^{(\mathrm{vac})}(r)=\sum_{\ell, m} f_{\ell m}^{(\mathrm{vac})}(r) Y_{\ell m}(\theta, \varphi)
$$

with

$$
f_{\ell m}^{(\mathrm{vac})}(r)=C_{\ell m}^{\prime} r^{\ell}+C_{\ell m} r^{-(\ell+1)}
$$

In these equations, $Y_{\ell m}(\theta, \varphi), j_{\ell}$ and $y_{\ell}$ are, respectively, the surface harmonics of the first kind and the spherical Bessel functions. Now, the coefficients $A_{\ell m}^{(i)}, B_{\ell m}^{(i)}, C_{\ell m}, C_{\ell m}^{\prime}, D_{\ell m}^{(i)}$ and $F_{\ell m}^{(i)}$ are subject to several constraints. These constraints include the requirement of no divergence of the potentials $\Phi^{(i)}$ for $r=0$ and $\Phi^{(\text {vac }}$ for $r \rightarrow \infty$ together with the boundary conditions (4)-(7). Using the well known properties of the surface harmonics, these boundary conditions amount to expressing continuity of the functions $f_{\ell m}^{(i)}(r)$ together with continuity of their derivatives at $R$ and $R+h$, and also continuity of the following functions $g_{\ell m}^{(i)}(r)$ at $R$ together with continuity of their derivatives at $R$ and $R+h$, where the functions $g_{\ell m}^{(i)}(r)$ are defined as

$$
g_{\ell m}^{(i)}=-\omega_{\mathrm{p}}^{(i) 2}\left[A_{\ell m}^{(i)} r^{\ell}+B_{\ell m}^{(i)} r^{-(\ell+1)}\right]-\omega^{2}\left[D_{\ell m}^{(i)} j_{\ell}\left(k^{(i)} r\right)+F_{\ell m}^{(i)} y_{\ell}\left(k^{(i)} r\right)\right] .
$$

The number of unknown coefficients determining the potentials $\Phi^{(1)}, \Phi^{(\text {II) }}$ and $\Phi^{\text {vac }}$ is ten: $A_{\ell m}^{(\mathrm{I})}, B_{\ell m}^{(\mathrm{I})}, D_{\ell m}^{(\mathrm{I})}, F_{\ell m}^{(\mathrm{l})}, A_{\ell m}^{(\mathrm{II})}, B_{\ell m}^{(\mathrm{II})}, D_{\ell m}^{(\mathrm{II})}, F_{\ell m}^{(\mathrm{I})}, C_{\ell m}$ and $C_{\ell m}^{\prime}$. On the other hand, the number of equations is also ten (two for the condition of no divergence of the potential at $r=0$, one for that at $r \rightarrow \infty$, four for continuity of the electric field and three for continuity of the electron current). Obviously, these equations are not linearly independent since (11) and (12) are linear and the potentials $\Phi^{(i)}$ determined up to a constant factor. Therefore, a non-trivial solution of the system is obtained, as usual, by expressing vanishing of its determinant, which yields the following rather complicated dispersion relation:

$$
X_{1}+X_{2}+X_{3}+X_{4}+X_{5}=0
$$


where

$$
\begin{aligned}
& X_{1}=\left(1-\left(k^{(\mathrm{II})} \beta^{(\mathrm{II})} / k^{(\mathrm{I})} \beta^{(\mathrm{I})}\right)^{2}\right)\left(k^{(\mathrm{l})} R j_{\ell}^{\prime}\left(k^{(\mathrm{I})} R\right)-\ell j_{\ell}\left(k^{(\mathrm{I})} R\right)\right)(R /(R+h))^{(\ell-1)} \\
& X_{2}=k^{(\mathrm{I})}\left(k^{(\mathrm{II})}(R+h)\right)^{3} j_{\ell}^{\prime}\left(k^{(\mathrm{I})} R\right) W_{1}\left(k^{(\mathrm{II})} R, k^{(\mathrm{II})}(R+h)\right) \alpha_{2} / k^{(\mathrm{II})} \\
& X_{3}=\left(k^{(\mathrm{II})}(R+h)\right)^{3} j_{\ell}\left(k^{(\mathrm{I})} R\right) W_{2}\left(k^{(\mathrm{II})} R, k^{(\mathrm{I})}(R+h)\right) \alpha_{2} \\
& X_{4}=\left(k^{(\mathrm{II})}(R+h)\right)^{2} j_{\ell}^{\prime}\left(k^{(\mathrm{I})} R\right) W_{3}\left(k^{(\mathrm{II})} R, k^{(\mathrm{II})}(R+h)\right) \alpha_{1} k^{(\mathrm{I})} / k^{(\mathrm{II})} \\
& X_{5}=-\left(k^{(\mathrm{II})}(R+h)\right)^{2} j_{\ell}\left(k^{(\mathrm{I})} R\right) W_{1}\left(k^{(\mathrm{II})} R, k^{(\mathrm{I})}(R+h)\right) \alpha_{1}
\end{aligned}
$$

and where we have let

$$
\begin{aligned}
& W_{1}(x, y)=-j_{\ell}(x) y_{\ell}^{\prime}(y)+y_{\ell}(x) j_{\ell}^{\prime}(y) \\
& W_{2}(x, y)=j_{\ell}^{\prime}(x) y_{\ell}^{\prime}(y)-y_{\ell}^{\prime}(x) j_{\ell}^{\prime}(y) \\
& W_{3}(x, y)=j_{\ell}(x) y_{\ell}(y)-y_{\ell}(x) j_{\ell}(y)
\end{aligned}
$$

with $j_{\ell}^{\prime}(x)=\partial j_{\ell}(x) / \partial x, y_{\ell}^{\prime}(x)=\partial y_{\ell}(x) / \partial x, \alpha_{2}=1+\left(k^{(\mathrm{II})} \beta^{(\mathrm{II})} / \omega\right)^{2}(\ell /(\ell+1))$ and $\alpha_{1}=\ell\left(\omega_{\mathrm{p}}^{(\mathrm{I})} / \omega\right)^{2}$. Since we are only interested in surface modes, it is understood that $k^{(\mathrm{I}) 2}<0$ and $k^{(\mathrm{II}) 2}<0$, that is, $k^{(\mathrm{I})}$ and $k^{(\mathrm{I})}$ are imaginary numbers.

For the radial part $E_{\mathrm{r}}$ of the self-consistent electric field $\left(E_{\mathrm{r}}(r)=\sum_{\ell, m} E_{\ell m}(r) Y_{\ell m}(\theta, \varphi)\right)$ in each medium, we easily obtain the following expressions:

$$
\begin{aligned}
& E_{\ell m}^{(\mathrm{I})}(r)=-A_{\ell m}^{(\mathrm{I})}\left[\ell r^{(\ell-\mathrm{I})}-\left(k^{(\mathrm{I})} \beta^{(\mathrm{I})} / k^{(\mathrm{I})} \beta^{(\mathrm{I})}\right)^{2} X_{6}\left(j_{\ell}^{\prime}\left(k^{(\mathrm{I})} r\right) / j_{\ell}^{\prime}\left(k^{(\mathrm{I})} R\right)\right)\right] \\
& E_{\ell m}^{(\mathrm{II})}(r)=-A_{\ell m}^{(\mathrm{I})}\left(k^{(\mathrm{l})} \beta^{(\mathrm{I})} / k^{(\mathrm{II})} \beta^{(\mathrm{I})}\right)^{2}\left\{\ell r^{(\ell-\mathrm{I})}+\left(k^{(\mathrm{II})}(R+h)\right)^{2}(R+h)^{(\ell-1)}\right. \\
& \left.\quad \times\left[\alpha_{1} W_{1}\left(k^{(\mathrm{II})}(R+h), k^{(\mathrm{II})} r\right)-k^{(\mathrm{II})}(R+h) \alpha_{2} W_{2}\left(k^{(\mathrm{II})} r, k^{(\mathrm{I})}(R+h)\right)\right]\right\}
\end{aligned}
$$

and

$$
E_{\ell m}^{\mathrm{vac}}(r)=-A_{\ell m}^{(\mathrm{l})}\left(k^{(\mathrm{l})} \beta^{(\mathrm{l})} / \omega\right)^{2} \ell(R+h)^{(\ell-1)}((R+h) / r)^{(\ell+2)}
$$

with

$$
\begin{aligned}
X_{6}=\ell R^{(\ell-1)}[- & \left.1+\left(k^{(\mathrm{I})} \beta^{(\mathrm{I})} / k^{(\mathrm{II})} \beta^{(\mathrm{II})}\right)^{2}\right]+\left(k^{(\mathrm{II})}(R+h)\right)^{2}(R+h)^{(\ell-1)} \\
& \times\left[k^{(\mathrm{II})}(R+h) \alpha_{2} W_{2}\left(k^{(\mathrm{II})} R, k^{(\mathrm{II})}(R+h)\right)-\alpha_{1} W_{\mathrm{I}}\left(k^{(\mathrm{II})}(R+h), k^{(\mathrm{II})} R\right)\right] .
\end{aligned}
$$

Relations (15)-(18) deserve to be commented on. Firstly, if we set $h=0$, taking account of the properties of the Wronskian $-W_{1}(x, x)$ of the Bessel functions and of the relation $W_{2}(x, x)=W_{3}(x, x)=0$, then relation (15) can be written

$$
j_{\ell}\left(k^{(\mathrm{I})} R\right)+(1 /(\ell+1))\left(1-((2 \ell+1) / \ell)\left(\omega / \omega_{\mathrm{p}}^{(\mathrm{I})}\right)^{2}\right) k^{(\mathrm{I})} R j_{\ell}^{\prime}\left(k^{(\mathrm{I})} R\right)=0
$$

which is recognized as the Crowell-Ritchie dispersion relation (Crowell and Ritchie 1968), as it should be. Besides, for the radial component of the self-consistent electric field, we find

$$
\begin{aligned}
& E_{\ell m}^{(\mathrm{I})}(r)=-A_{\ell m}^{(\mathrm{l})}\left[\ell r^{(\ell-1)}-\ell R^{(\ell-1)}\left(\omega_{\mathrm{p}}^{(\mathrm{l})} / \omega\right)^{2}\left(j_{\ell}^{\prime}\left(k^{(\mathrm{I})} r\right) / j_{\ell}^{\prime}\left(k^{(\mathrm{I})} R\right)\right)\right] \\
& E_{\ell m}^{\mathrm{vac}}(r)=-A_{\ell m}^{(\mathrm{I})}\left(k^{(\mathrm{I})} \beta^{(\mathrm{I})} / \omega\right)^{2} \ell R^{(\ell-1)}(R / r)^{(\ell+2)} .
\end{aligned}
$$

Firstly, for $\ell=1, m=0$, in the limit $k^{(\mathrm{I})} R \rightarrow \infty$, the classical sp frequency $\omega=\omega_{\mathrm{p}}^{(\mathrm{l})} / \sqrt{3}$ is immediately recovered from $\left(15^{\prime}\right)$. Secondly, if we take $n_{0}^{(\mathrm{I})}=n_{0}^{\text {(II) }}$ (trivial case), the term $X_{1}$ cancels out, as is the case of $X_{2}+X_{3}$. We then meet again the Crowell-Ritchie dispersion relation $\left(15^{\prime}\right)$ for a medium of density $n_{0}^{(\mathrm{II})}$ and of radius $R+h$, as we should do. In the general case, the SP frequency and the radial part of the field are respectively given by the rather cumbersome expressions (15) and (16)-(18), which, however, lend themselves to an easy numerical investigation. 


\section{Results and interpretation}

For the sake of simplicity, the numerical studies have been performed for the first harmonic case $(\ell=1, m=0)$ (actually, as in the Jensen situation (one metallic medium), the case $(\ell=0, m=0)$ does not need to be considered since the potentials $\Phi^{(i)}$ reduce to a constant) for various free-electron metal systems such as $\mathrm{Al}-\mathrm{Cu}, \mathrm{Na}-\mathrm{K}$ and $\mathrm{Cs}-\mathrm{Rb}$. Similar tendencies have been observed in those systems and, in the present paper, we shall examine systematically the particular couple $\mathrm{K}-\mathrm{M}$, where $\mathrm{M}$ denotes a fictitious free-electron metal of density $\frac{1}{2} n_{0}^{(\mathrm{K})}$. As we shall see, the interest of this model is to enable us to give some comments on the role of ion- and electron-density inhomogeneities.

\section{$\omega(\mathrm{rad} / \mathrm{s})$}

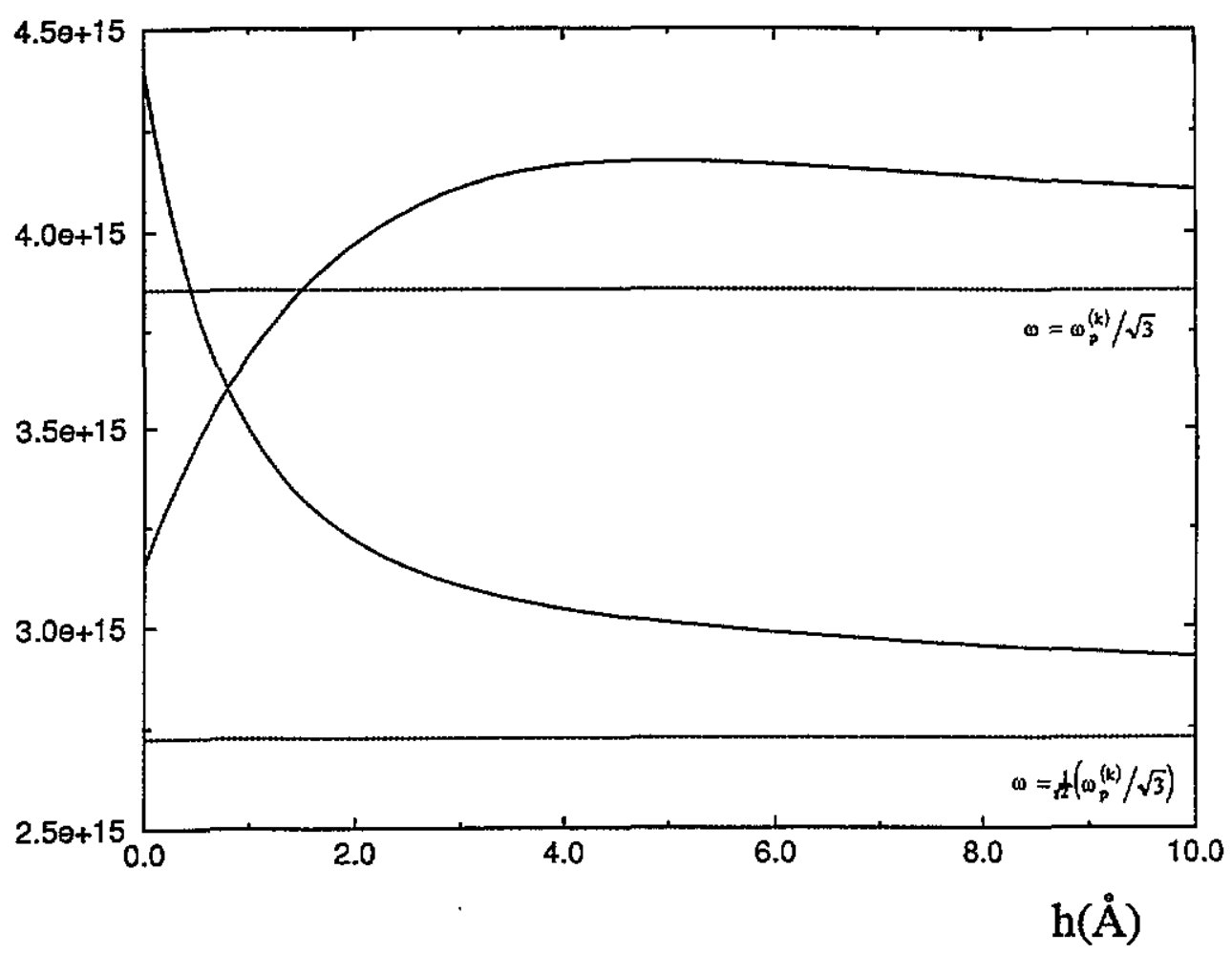

Figure 1. SP frequencies for the system $\mathrm{K} / \mathrm{M}$ (upper curve) and for the system $\mathrm{M} / \mathrm{K}$ (lower curve) versus $h$ for $R=10 \AA$ (M denotes a fictitious free-electron metal of density $\frac{1}{2} n_{0}^{(K)}$ and $\omega_{\mathrm{p}}^{(\mathrm{K})}=6.67 \times 10^{15} \mathrm{rad} \mathrm{s}^{-1}$ (from Ashcroft and Mermin 1976)).

In the computations, three typical values of the bulk radius, $R=10 \AA, 100 \AA$ and $1000 \AA$, together with various layer thickness $h$ (up to $10 \AA$ ) have been considered. A remarkable characteristic feature is the small range over which a rapid variation of the SP frequency $\omega$ is obtained (cf figures 1-3). As can be seen, there exists a range of thickness where the SP frequency is strongly different from the classical values $\omega_{\mathrm{p}}^{(\mathrm{I})} / \sqrt{3}$ and $\omega_{\mathrm{p}}^{(\mathrm{II})} / \sqrt{3}$. Reversing the role of the two metals yields symmetrical behaviours. These features can be interpreted as typical size effects: for a simple (uncoated) system, the value of the SP 


\section{$\omega(\mathrm{rad} / \mathrm{s})$}

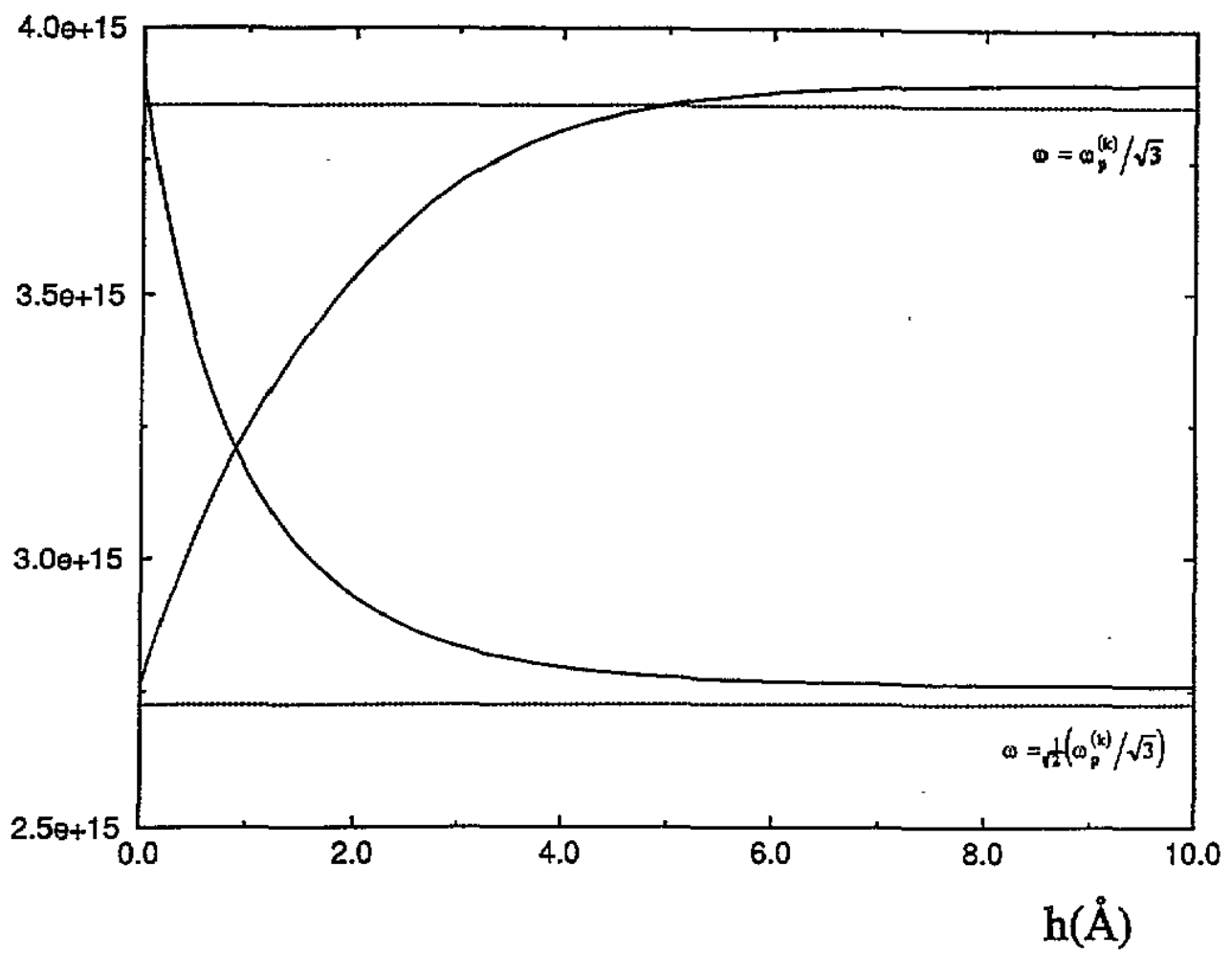

Figure 2. The same as figure $l$ but for $R=100 \AA$.

frequency is not $\omega_{\mathrm{p}} / \sqrt{3}$ but rather $\omega_{\mathrm{p}} / \sqrt{\alpha}$ where $\alpha<3$ and tends toward this value when $k^{(\mathrm{I})} R \rightarrow \infty$. This result just stems from the original Crowell-Ritchie dispersion relation $\left(15^{\prime}\right)$. In this relation, the Bessel functions express the influence of opposite surfaces. When the size of the sphere is large enough $\left(R \gg k^{(\mathrm{l})-1}\right)$, these influences play a negligible role and $\alpha$ tends towards the asymptotic value $\alpha=3$ as a consequence of the asymptotic behaviour of the Bessel functions. Now, for a coated sphere, two types of size effect need therefore to be considered: (i) those connected with the size of the whole system (medium (I) plus medium (II)), which are of the same nature as those described for an uncoated system, and (ii) those connected with the layer thickness, which represent interactions between interfaces of a different kind (i.e. metal-metal and metal-vacuum). Hence, the values $\omega_{\mathrm{p}}^{(\mathrm{I})} / \sqrt{3}$ (bulk regime) and $\omega_{\mathrm{p}}^{a)} / \sqrt{3}$ (layer regime) appear as limit values: the bulk regime is defined when $R / h \gg 1$ and $h \ll k^{(\mathrm{II})-1}$ and the layer regime when $h \gg k^{(\mathrm{II})-1}$.

As mentioned above, the present two-layer system may also give useful indications about the part played for collective surface modes by the ion density profile near the surface of small metal clusters. Let us consider, for example, a linear ion density profile. As would be done in a numerical treatment of this problem, such a profile may be simulated by a sequence of small steps, each one being considered as a homogeneous layer (see e.g. Boardman and Paranjape 1977, Boardman 1982). As a multiple-step analysis is beyond the scope of the present paper, we shall only consider here the influence of one single layer and we will 


\section{$\omega(\mathrm{rad} / \mathrm{s})$}

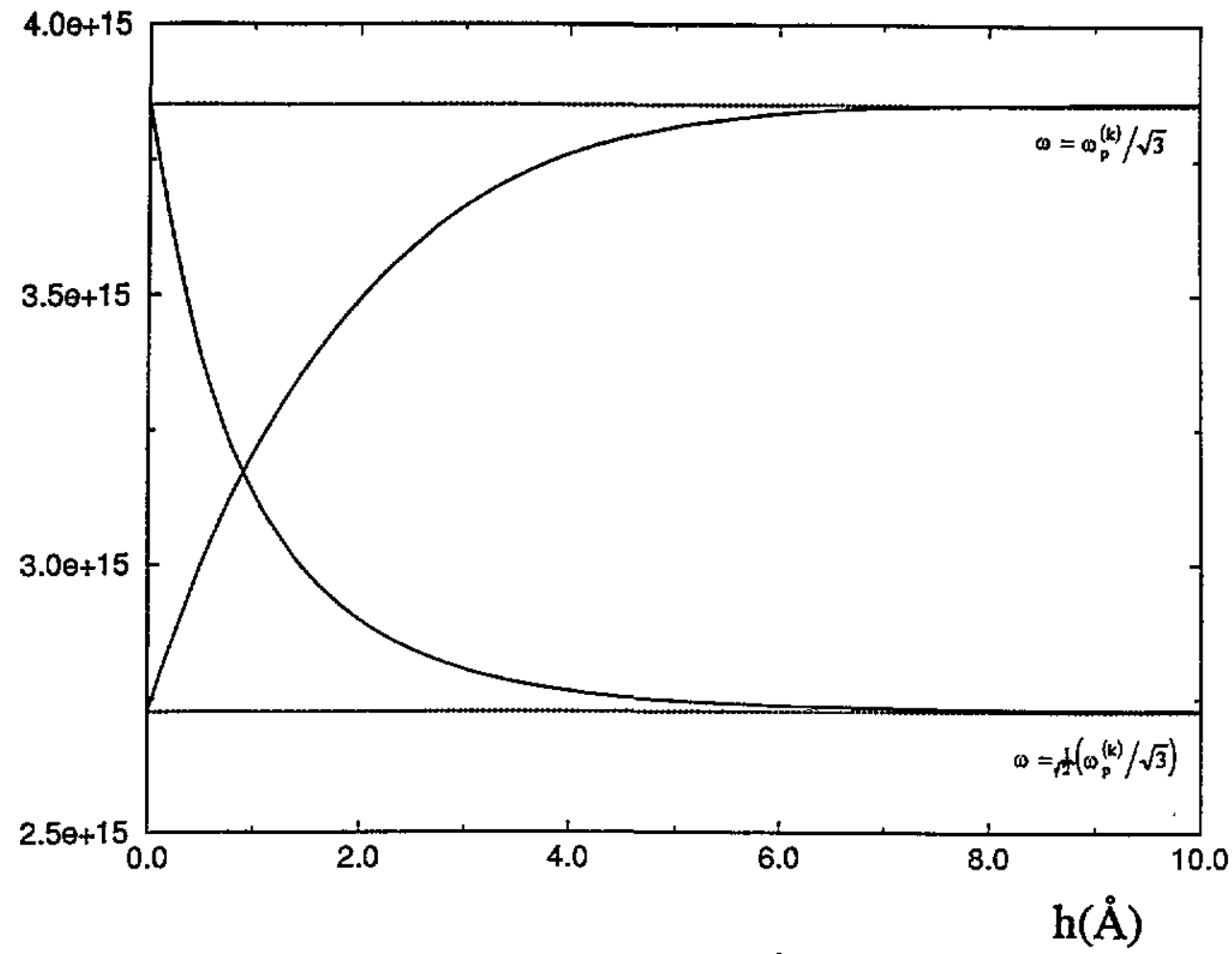

Figure 3. The same as figure 1 but for $R=1000 \AA$.

simulate a linear ion density profile of the form $n_{0}[1-(R-r) / h]$ with $R \leqslant r \leqslant R+h$, by a single-step profile of height $\frac{1}{2} n_{0}$ and of length $h$, as has been considered in figures $1-3$. This approximation will be sufficient, however, for a qualitative estimate of the influence of an ion density gradient and an electron density gradient. As is clearly exhibited in figures $1-3$, the influence of the layer is to induce a red shift of the SP frequencies (see the remark below, however). It is interesting to note that this effect, which is a general tendency for freeelectron metals and which was also noted by Ruppin (1976) and Boardman and Paranjape (1977), has been experimentally observed. However, it should also be emphasized that the applicability of the hydrodynamical approach in the cases of very sharp density profiles or surface diffuseness simulated by a very thin layer corresponding to a few atomic layers is certainly questionable, as is the validity of the jellium approximation. As shown above, this situation just tallies with the domain where the plasmon frequencies shift abruptly. A microscopic investigation (see e.g. Brack 1993) of this effect is therefore unavoidable and would complete the present macroscopic analysis, particularly for small systems such as metal clusters where singular effects have been recently revealed (Bréchignac et al 1993). Furthermore, it should be kept in mind that the present study only holds for simple metals and cannot be considered in the case of other metals such as transition metals (e.g. Ag) where blue shifts have actually been observed. For transition metals, interband transitions play an essential part that cannot be accounted for in a free-electron-gas model (Liebsch 1993). 


\section{$\mathrm{E}_{\mathrm{r}}$ (a.u.)}

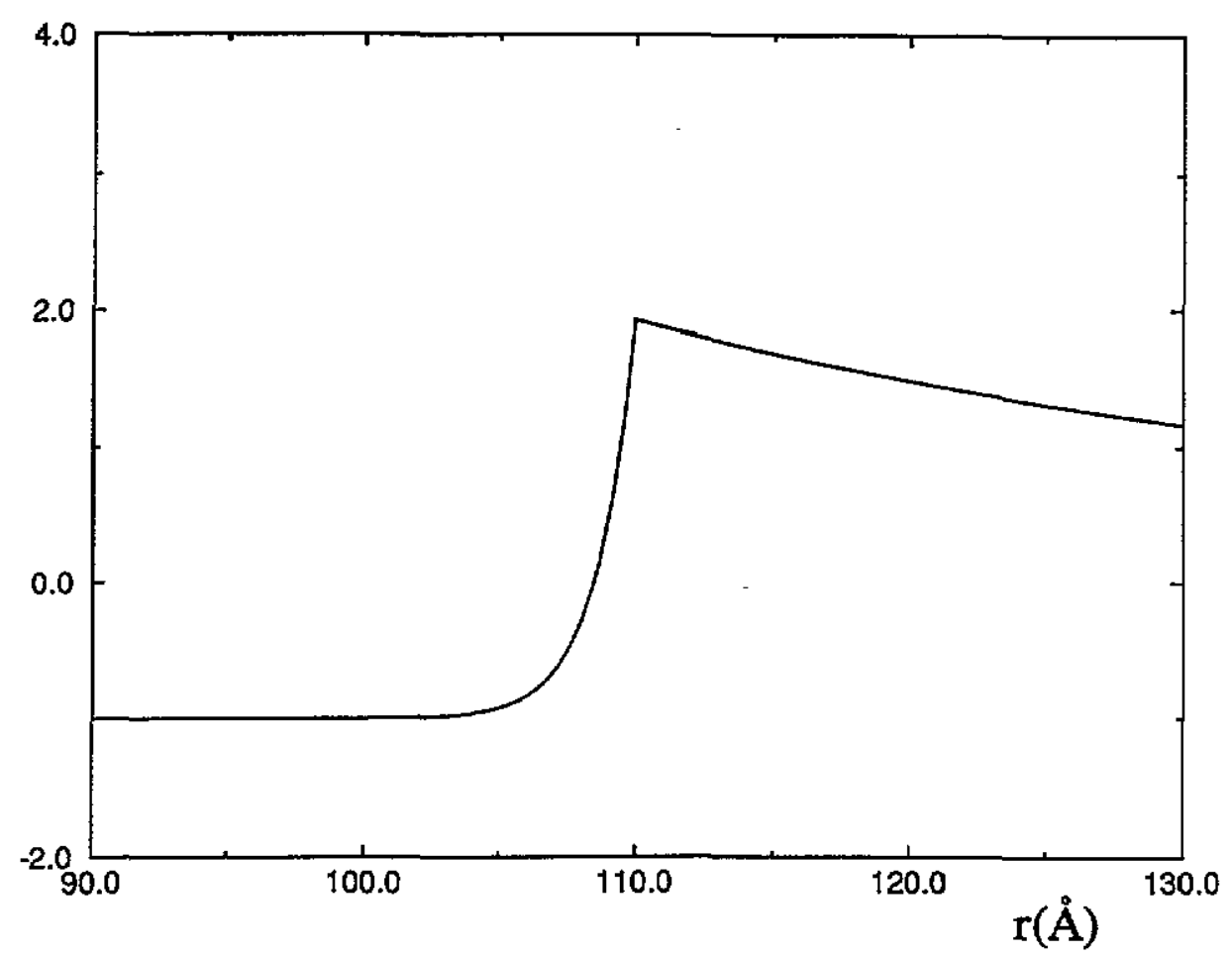

Figure 4. The radial component of the self-consistent electric field at $t=0$ for $\mathrm{K}$ and $R=100 \AA$ versus $r$ (the Jensen case).

In figures 4-6 are reported (in arbitrary units) the amplitude variations of the radial part of the self-consistent electric field. For $h=0$ (the uncoated metal case, see figure 4), the well known resonance electric field of the SP is represented and shows the usual spatial variations of the field amplitude inside the metal bulk and in vacuum. For $h=10 \AA$ (see figures 5 and 6), the self-consistent electric field exhibits a strong enhancement at the metal-metal interface (at $r=R=100 \AA$ ). This field is characterized by an amplitude much greater than that of the surface plasmon (at $r=R+h=110 \AA$ ). It is important to emphasize that this interface plasmon has the same frequency as the surface plasmon since it is found by solving the dispersion relation (15). Actually, this new effect, which represents strong charge oscillations at the interface, admits a simple physical interpretation.

By combining (8), (9) and (10), we can easily rewrite the linearized Poisson equation in the following form:

$$
\nabla \cdot E^{(l)}=\nabla \cdot\left[\left(4 \pi e^{2} / m \omega^{2}\right) n_{0}^{(i)} E^{(i)}+\left(4 \pi e / m \omega^{2}\right) \nabla P^{(l)}(r)\right] .
$$

Neglecting the kinetic-pressure term between the square brackets (that is, neglecting any dissipation process), this equation becomes nothing else than

$$
\nabla \cdot D^{(i)}=0
$$

where

$$
\boldsymbol{D}^{(i)}=\varepsilon^{(i)} \boldsymbol{E}^{(i)}
$$




\section{Er(a.u.)}

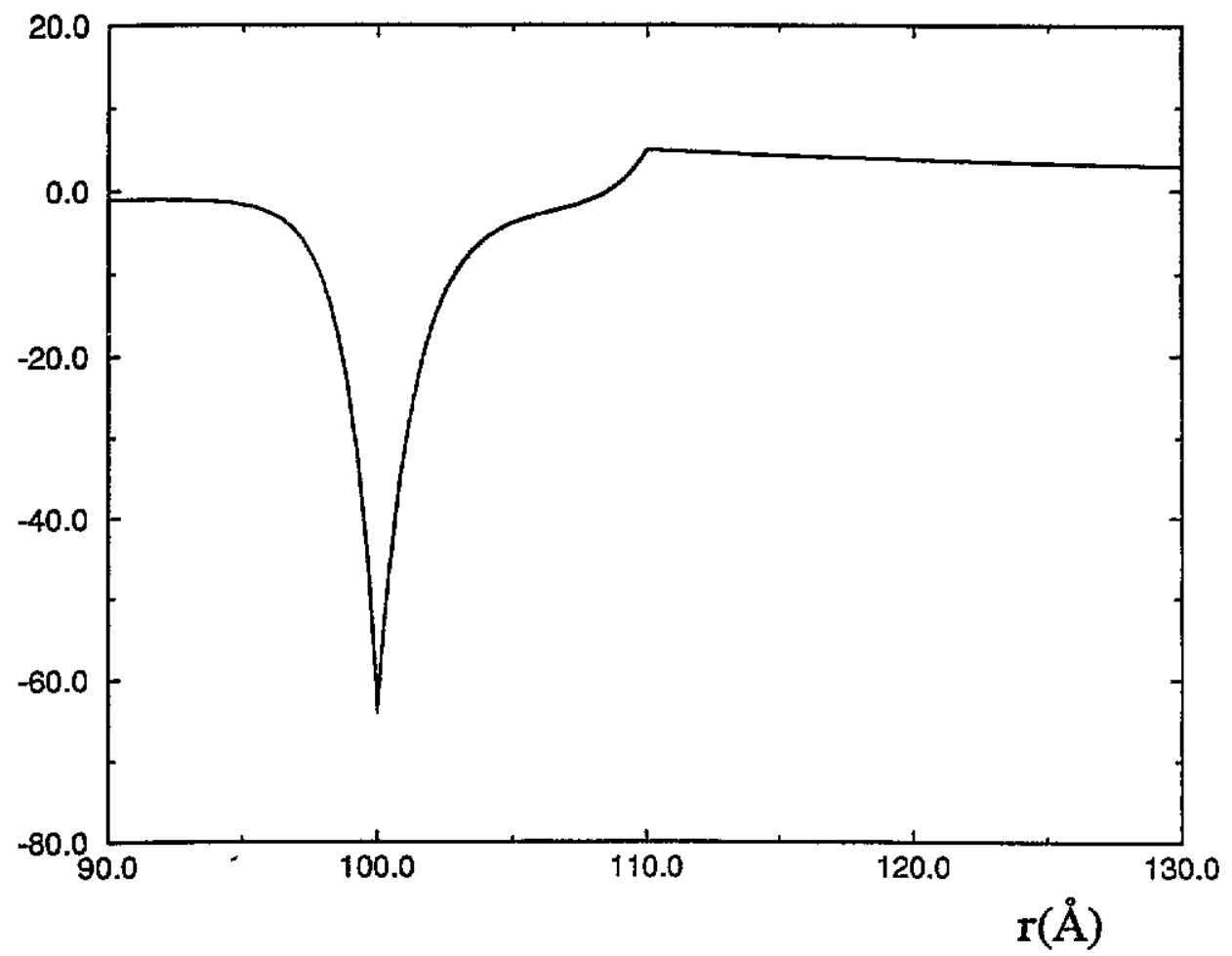

Figure 5. The radial component of the self-consistent electric field at $t=0$ for the system $\mathrm{K} / \mathrm{M}$ and $R=100 \AA$ versus $r$.

is the electric displacement and

$$
\varepsilon^{(i)}=1-\omega_{\mathrm{p}}^{(i) 2} / \omega^{2}
$$

is the plasma dielectric function for each medium (i). Now, (19) can also be rewritten as

$$
\nabla \cdot E^{(i)}=-\left(\nabla \varepsilon^{(i)}\right) \cdot E^{(i)} / \varepsilon^{(i)}
$$

Taking (8) into account, this equation can finally be expressed in the form

$$
n^{(i)}=-\left(e / m \omega^{2}\right)\left(\nabla n_{0}^{(i)}\right) \cdot E^{(i)}(\omega) / \varepsilon^{(i)}(\omega)
$$

which shows that, at the metal-metal interface where the charge density varies abruptly from $n_{0}^{(\mathrm{I})}$ to $n_{0}^{(\mathrm{II})}$, important charge fluctuations may occur, depending on whether there exists a radial component of the electric field at this place. This electric field can be just that of an SP existing at the edge of the metal layer. Provided the layer thickness is thin enough, the field of the SP tunnels into the metal-metal interface and so enhances the charge fluctuations at the same frequency as the SP. The magnitude of the enhanced field is then limited by thermal effects that are accounted for by the kinetic-pressure term (and by electron-electron collisions that are not considered here, however). 


\section{Er(a.u.)}

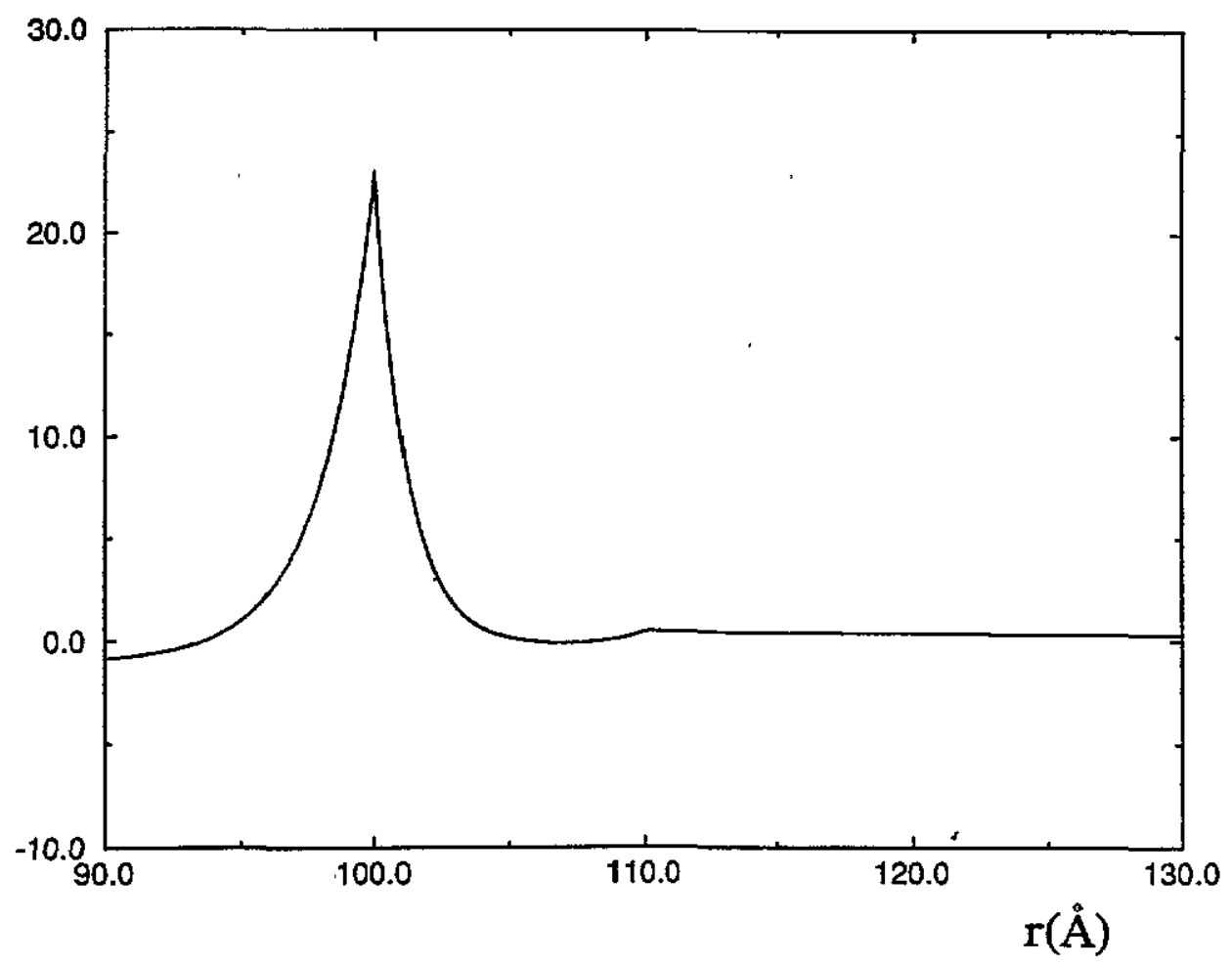

Figure 6. The same as figure 5 but for the system $\mathrm{M} / \mathrm{K}$.

\section{Conclusion}

In this paper, we have given an exact generalization of the Bloch-Jensen hydrodynamical model for free-electron metals to a two-layer spherical system. This system has been proven useful to show that the ion density profile near the surface of a small metal cluster plays an important part inasmuch as it can result in significant shifts of the SP frequencies. This tendency would need to be corroborated by a microscopic analysis going beyond the jellium device together with further investigations involving a multiple-step formalism taking the exact ion density profile into consideration. Besides, we have taken advantage of the generalization to show an interesting effect: the existence of metal-metal interface plasmons enhanced by the SPS. This effect would also deserve to be investigated in planar geometry for which experiments are more easily feasible. Also, the consequences of electron-electron interactions should be weighed up as they are dissipative effects. In fact, their influence is probably to modify the above form of the kinetic pressure. We hope to answer these questions in a future paper.

\section{Acknowledgment}

The authors acknowledge with thanks Mrs $N$ Auby for her help in the numerical computations. 


\section{Appendix}

We give here a demonstration of validity of (2), taking the surface discontinuities into account (for the fluid equation of motion and the Poisson equation, the proof is in fact trivial).

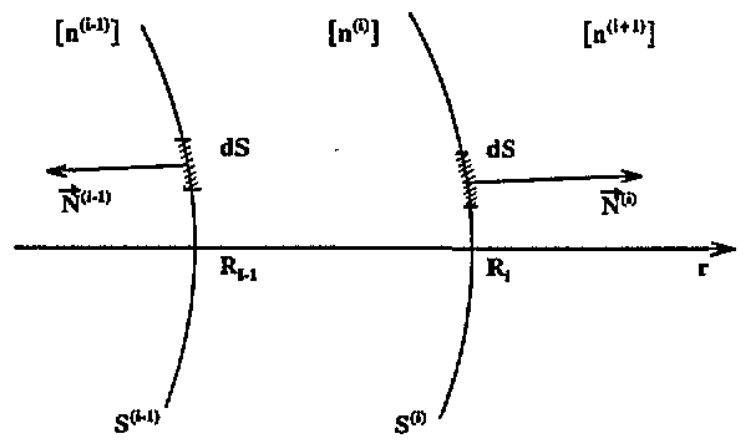

Figure A1. A schematic representation of our notations.

Let us consider a small volume $V^{(i)}$ included between two spherical sheets located at $R_{i-1}$ and $R_{i}$ such that the electron density and velocity are respectively $n^{(i-1)}$ and $v^{(i-1)}$ for $r<R_{i-1}, n^{(i)}$ and $v^{(i)}$ for $R_{i-1}<r<R_{i}$ and $n^{(i+1)}$ and $v^{(i+1)}$ for $r>R_{i}$. The number of electrons in volume $V^{(i)}$ at time $t$ is then $\int_{V^{(i)}} n^{(i)} \mathrm{d} V$, where $\mathrm{d} V$ is an element of volume. The variation per unit time of this number is $\partial \int_{V^{(i)}} n^{(i)} \mathrm{d} V / \partial t$, which can be also obtained, as usual, by drawing up a balance between the number of incoming and outgoing electrons in $V^{(i)}$. If $S^{(i)}$ denotes the surface of the sphere of radius $R_{i}$ and $N^{(i)}$ a normally oriented unit vector of this surface (see figure A1), $n^{(i)} v^{(i)} \cdot N^{(i)}$ represents the electron flow through the surface element $\mathrm{d} S$ of $S^{(i)}$. We can thus write

$$
\partial \int_{V^{(i)}} n^{(i)} \mathrm{d} V / \partial t=T_{1}+T_{2}+T_{3}+T_{4}
$$

where

$$
\begin{aligned}
& T_{1}=-\oint_{S^{(i)}} n^{(i+1)} v^{(i+1)} \cdot N^{(i)} \Theta\left(-v^{(i+1)} \cdot N^{(i)}\right) \mathrm{d} S \\
& T_{2}=-\oint_{S^{(i-1)}} n^{(i-1)} v^{(i-1)} \cdot N^{(i-1)} \Theta\left(-v^{(i-1)} \cdot N^{(i-1)}\right) \mathrm{d} S \\
& T_{3}=-\oint_{S^{(i)}} n^{(i)} v^{(i)} \cdot N^{(i)} \Theta\left(-v^{(i)} \cdot N^{(i)}\right) \mathrm{d} S \\
& T_{4}=-\oint_{S^{(i-1)}} n^{(i)} v^{(i)} \cdot N^{(i-1)} \Theta\left(-v^{(i)} \cdot N^{(i-1)}\right) \mathrm{d} S
\end{aligned}
$$

where $\Theta(x)$ is the Heaviside function and where the $T_{1}$ and $T_{2}$ terms represent the incoming electron flow in $V^{(i)}$ and the $T_{3}$ and $T_{4}$ terms the outgoing one. Now, on each surface $S^{(i)}$, we have a density discontinuity. If we impose continuity of the normal (here radial) component of the electron current density $j=-e n v$ through $S^{(i)}$ and $S^{(i-1)}$, that is

$$
\left[j^{(i)} \cdot N^{(i)}\right]_{S^{(i)}}=\left[j^{(i+1)} \cdot N^{(i)}\right]_{S^{(i)}}
$$


and

$$
\left[j^{(i)} \cdot N^{(i-1)}\right]_{S^{(t-1)}}=\left[j^{(i-1)} \cdot N^{(i-1)}\right]_{S^{(i-1)}}
$$

we can regroup in (A1) the $T_{1}$ and $T_{2}$ terms and also the $T_{3}$ and $T_{4}$ ones, making use of the fact that the sign of $v^{(i)} \cdot N^{(i)}$ is the same as that of $j^{(i)} \cdot N^{(i)}$ and using the relation $\Theta(x)+\Theta(-x)=1$. Then, (A1) becomes

$\partial \int_{V^{(i)}} n^{(i)} \mathrm{d} V / \partial t=-\oint_{S^{(i)}} n^{(i)} v^{(i)} \cdot N^{(i)} \mathrm{d} S-\oint_{S^{(i-1)}} n^{(i)} v^{(i)} \cdot N^{(i-1)} \mathrm{d} S$.

Now, as $N^{(i-1)} \mathrm{d} S=-N^{(i)}$ (see figure 3) and using the Ostrogradsky theorem, (A2) can be rewritten as

$$
\partial \int_{V^{(i)}} n^{(i)} \mathrm{d} V / \partial t=-\int_{V\left(S^{(i)}\right)} \nabla \cdot\left(n^{(i)} v^{(i)}\right) \mathrm{d} V+\int_{V\left(S^{(i-1)}\right)} \nabla \cdot\left(n^{(i)} v^{(i)}\right) \mathrm{d} V
$$

where $V\left(S^{(i)}\right)$ and $V\left(S^{(i-1)}\right)$ are the volumes of the spheres of radius $R_{i}$ and $R_{i-1}$, respectively. Finally, as $V^{(i)}=V\left(S^{(i)}\right)-V\left(S^{(i-1)}\right)$, we have

$$
\partial \int_{V^{(i)}} n^{(i)} \mathrm{d} V / \partial t=-\int_{V^{(i)}} \nabla \cdot\left(n^{(i)} v^{(i)}\right) \mathrm{d} V
$$

which leads to the familiar equation of continuity in medium (i)

$$
\partial n^{(i)}(r, t) / \partial t+\nabla \cdot\left[n^{(i)}(r, t) v^{(i)}(r, t)\right]=0
$$

In summary, validity of the equation of continuity in medium (I) and (II) depends on the condition of continuity of the normal (radial) component of the electron current density at the metal-metal and metal-vacuum interfaces. It is worth noticing that continuity of the normal components of the electron current density $j$ and of the electric field $E$ ensures continuity of the normal component of the electric displacement $D$ defined by the equation

$$
\partial D / \partial t=\partial E / \partial t+4 \pi j
$$

which can be written in the present case as

$$
D=E+(4 \pi \mathrm{i} / \omega) j
$$

The standard electrostatic boundary conditions (continuity of the potential and of the electric displacement) are thus satisfied as a. special case.

\section{References}

Ashcroft N W and Mermin N D 1976 Solid State Physics (Philadelphia, PA: CBS)

Bloch F 1933 Z. Phys. 81363

Boardman A D 1982 Electromagnetic Surface Modes ed A D Boardman (New York: Wiley) p 41

Boardman A D and Paranjape B V 1977 J. Phys. F: Met. Phys. 71935

Brack M 1993 Rev. Mod. Phys. 65677 and references therein

Bréchignac C, Cahuzac P, Leygnier J and Sarfati A 1993 Phys. Rev. Lett. 702036

Courant R and Hilbert D 1989 Methods of Mathematical Physics vol I (New York: Wiley) 
Crowell J and Ritchie R H 1968 Phys. Rev. 172436

De Heer W A 1993 Rev. Mod. Phys. 65611 and references therein

Jackson J D 1976 Classical Electrodynamics 2nd edn (New York: Wiley)

Jensen H 1937 Z. Phys. 106620

Liebsch A 1993 Phys. Rev. 4811317 and references therein

Lipparini E and Pederiva F 1993 Z Phys. D 27281

Lundqvist S 1983 Theory of the Inhomogeneous Electron Gas ed S Lundqvist (New York: Plenum) p 149

Malov Y A and Zaretsky D F 1993 Phys. Lett. 177A 379

Parks J H and McDonald S A 1989 Phys. Rev. Lett. 622301

Pines D 1964 Elementary Excitation in Solids (New York: Benjamin)

Ruppin R 1976 J. Opt. Soc. Am. 66449

Wang Y, Lewenkopf C, Tomanek D and Bertsch G 1993 Chem. Phys. Lett. 205521

Xu M and Dignam M J 1992 J. Chem. Phys. 963370 